\title{
Research on Accumulator Charging Model in UPS
}

\author{
Zheng Liu, Huihui Zou, Bo ZHANG \\ China Satellite Tracking and Controlling Department, Jiangyin 214431, China \\ liuzheng354@qq.com
}

Keywords: UPS, accumulator, model

Abstract. UPS (Uninterruptible Power System) is a kind of high-powder electronic device that can provide high-quality electric power continuously and stably. Accumulator plays important role as energy storage equipment and its charging and discharging process is a complex electrochemical reaction, and parameters have strong nonlinearity and discreteness. At present, there is no unified and accurate method to carry out modeling and simulation to accumulator system and model difference of every kind of battery is large; therefore, it is necessary to develop model with small error and less calculated amount and with change performance consistent with accumulator in actual working condition.

\section{Introduction}

Accumulator is important energy storage equipment for UPS system to provide power continuously and improper usage and maintenance will influence performance of accumulator and shorten its life; therefore, a set of effective accumulator model shall be established to ensure healthy condition of accumulator in UPS system. Researches of accumulator in modeling begins at 1950s, extensive researches in the field have been carried out at abroad and a large number of achievements have been obtained and many science and research institutions have developed many accumulator simulation software and procedures, such as ADVISOR[1] (Advanced Vehicle Simulator) developed by National Renewable Energy Laboratory and PSATC (Powertrain System Analysis Toolkit) developed by National Laboratory set in the University of Chicago, etc.

\section{Classification of model}

Accumulator models can be classified into the following kinds from the purpose of researching model.

Mechanism model. This kind of model is to better help researchers to understand physical, chemical and electrical phenomenon in charging and discharging processes. It focuses on considering characteristics of counter electrode and reaction rate of different composition materials to improve electrode and electrolyte material, which is used for development of battery industry. Researchers of University of Pennsylvania have mastered solid-state physics characteristics, structure of reaction junction face and chemical reaction of electrode material and have put forward micro-macro model by adopting computational fluid mechanics method and the model has researched reactions of all materials under on-equilibrium conditions of interface and can better describe characteristics of electrochemistry power supply of high-energy density and high-power density and high accuracy of this kind of model is required during micro to macro transformation process and numerical analysis technology and partial differential equation are required and therefore, calculated amount is large.

External-characteristic parameter model. This kind of model is established by people to forecast external electric parameters after adopting fixed charging and discharging methods. Changes of voltage-time, discharge resistance and electric quantity can be observed by utilizing this kind of model. This kind of model mainly includes temperature model, SOC (State of Charge) model, cycle life model and capacity fading model, etc[2].

Equivalent circuit model. Because physical, chemical and electric phenomenon of accumulator for mechanism model shall be considered during charging and discharging process of accumulator, this kind of model is usually very complex. To describe dynamic characteristics of unit cell and consider 
practicability in electric engineering, it is put forward that double-layer electrode and diffusion phenomenon can be stimulated by equivalent circuit. Characteristics of electrochemistry and influence of temperature are considered simultaneously and dynamic performance of battery can be forecasted accurately, mainly including basic circuit model, linear circuit model and nonlinear circuit model, etc.

\section{Equivalent circuit model and application analysis}

Main purpose of establishing accumulator model at home and abroad is identification, state supervising and performance test of certain state parameter of accumulator. The paper puts forward the research to apply equivalent circuit model of accumulator to quick charging method. Under current situation of accumulator model research, equivalent circuit model has been widely researched and applied with the characteristics of clear dynamic characteristics and strong practicability. Existing equivalent circuit model mainly includes basic model, Thevenin model, four-order dynamic model and improved model, etc.

\section{Basic model.}

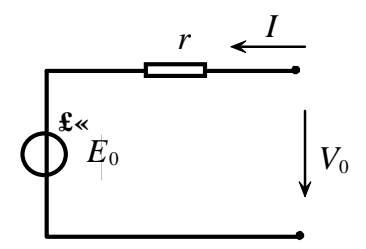

Fig. 1 Basic Model

Fig. 1 is common battery model [3]. The model consists of an ideal battery (electromotive force is $E_{0}$ ) and an equivalent internal resistance $r$ and $V_{0}$ is terminal voltage of battery and $I$ is electric current flowing past battery. It can be acquired according to Ohm's law of whole circuit:

$$
I=\frac{V_{0}-E_{0}}{r}
$$

Although the model is common, change of internal battery resistance caused by electrically charged state of battery, concentration change of electrolyte solution and sulfate generation is not considered. The model is only applicable to assumption condition where infinite power can be obtained from battery or electrically charged state of battery is not important.

Improved model. Jean Paul Cun has put forward improved model based on primary model in Fig.1. In the model, internal resistance $r$ is not constant, but variable changing with electrically charged state of battery; electrically charged state of battery is considered in model. Assumed that:

$$
r=\frac{R_{0}}{S O C^{k}}
$$

Where, $R_{0}$ is initial resistance of battery; $\mathrm{SOC}=1-A h / C_{10}$ and $C_{10}$ is 10 Ah under standard battery temperature; $A h$ is discharge capacity; range of variation of $S O C$ is $0 \sim 1$; $\mathrm{k}$ is coefficient and it is function of discharge rate.

Thevenin battery model. Thevenin model is another kind of common accumulator model. The model is composed of ideal power supply $E_{0}$, internal resistance $\mathrm{r}$, capacitance $C_{0}$ and overvoltage resistance $R_{0}$, as shown in Fig.2. In the figure, $C_{0}$ represents capacitances between parallel counter electrodes; $R_{0}$ represents nonlinearity contact resistance between counter electrode and electrolyte. Assumed that voltage $u_{C 0}$ in both sides of capacitance $C_{0}$ is state variable, it can be gotten according to circuit law that:

$$
r \cdot C_{0} \frac{\mathrm{d} u_{C_{0}}}{\mathrm{~d} t}+\left(1+\frac{r}{R_{0}}\right) u_{C_{0}}=V_{0}-E_{0}
$$




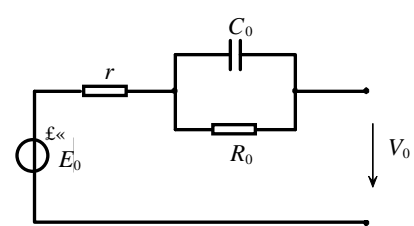

Fig. 2 Thevenin Battery Model

Main defect of the model is that all parameters in model are set as constant, while these parameters are all functions of battery state actually.

Four-order dynamic model.People, such as Gigli have come up with a kind of dynamic model, as shown in Fig. 3. Dynamic model is composed of two parts:

Electric current $I_{\mathrm{p}}$ passes through $R_{\mathrm{p}}, R_{\mathrm{d}}, C_{\mathrm{b}}, R_{\mathrm{w}}$ and $C_{\mathrm{w} .} R_{\mathrm{p}}$ is resistance parameter relevant to electrolyte reaction; $R_{\mathrm{d}}$ is parameter relevant to function of Ohm internal resistance; $C_{\mathrm{b}}$ is drain capacitance parallel with $R_{\mathrm{d}} ; R_{\mathrm{w}}$ is parameter relevant to energy loss and $C_{\mathrm{w}}$ is drain capacitance parallel with $R_{\text {w. }}$

Self-discharge electric current $I_{\mathrm{s}}$ passes through $R_{\mathrm{s}}$. The model has the defects of complex structure and many identification parameters.

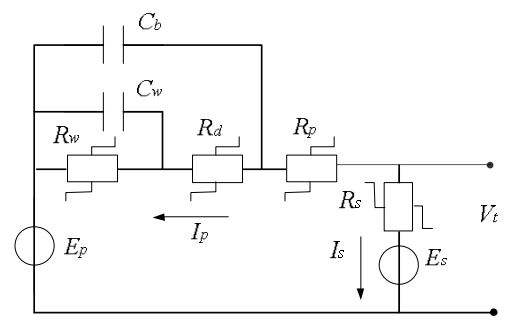

Fig. 3 Four-order Dynamic Model

Primary model and Thevenin model only involve parameters of internal resistance and electrically charge state, while variation of temperature and polarization in charging process of accumulator is not considered. Therefore, characteristics of accumulator cannot be reflected comprehensively in charging process of accumulator. Four-order dynamic model has considered main-reaction and side-reaction process in charging process from charging energy flow direction directions, which conforms to actual electrochemical reaction process of accumulator. However, because it involves many parameters and orders of model are relatively high, it brings many difficulties to parameter identification, which is adverse to practical application.

\section{Model parameters identification and state estimation method}

Existing parameter identification methods include numerical analysis method, empirical formula method, neural network method and Kalma filtering method, etc. Realization of above methods requires establishing relationship model between model parameter and measurable parameter based on experience, acquiring measurable data of model and adopting identification algorithm.

Parameter identification method is usually combined with parameter optimization algorithm. Common parameter optimization algorithm includes: genetic algorithm[4], method of exhaustion and $\mathrm{K}-\mathrm{CV}$ cross validation method. Besides, there are algorithms of some simulated annealing algorithm, tabu search, artificial ant swarm algorithm and particle swarm optimization algorithm, etc. Besides above identification method of model parameters, parameter identification can be carried out according to data provided by battery manufacturers. In these data, the most important is data relevant to battery capacity[5].

\section{Conclusions}

Establishing accurate accumulator model can manage and optimize charging and discharging process of accumulator to better maintain and repair accumulator. Besides, in design and production process of accumulator, establishing model can help designers to optimize design scheme, shorten design period 
and improve design quality. When researching intelligent charging method, experiment cost can be reduced by modeling and more accurate data can be obtained.

\section{References}

[1] $\mathrm{Wu} \mathrm{B}$, Dougal R, White R E. Resistive companion battery modeling for electric circuit simulations[J]. Journal of Power Sources, 2001, 93(1-2):186-200.

[2] Tenno A, Tenno R, Suntio T. Battery impedance and its relationship to battery characteristics[C]// Telecommunications Energy Conference, 2002. Intelec. International. IEEE Xplore, 2002:176-183.

[3] Szumanowski A, Chang Y. Battery Management System Based on Battery Nonlinear Dynamics Modeling[J]. IEEE Transactions on Vehicular Technology, 2008, 57(3):1425-1432.

[4] Deb K, Pratap A, Agarwal S, et al. A fast and elitist multiobjective genetic algorithm: NSGA-II[J]. IEEE Transactions on Evolutionary Computation, 2002, 6(2):182-197.

[5] Rong P, Pedram M. An analytical model for predicting the remaining battery capacity of lithium-ion batteries[J]. IEEE Transactions on Very Large Scale Integration Systems, 2006, 14(5):441-451. 